\title{
Distally Based Medial Hemisoleus Muscle Flap for Reconstruction of Distal and Middle Third Leg Defects
}

\author{
HELMY ELWAKEEL, M.D. \\ The Department of Plastic Surgery, Faculty of Medicine, Alexandria University, Egypt
}

\begin{abstract}
Background: Coverage of distal leg defects is quite challenging. Usually, free tissue transfer is used for the reconstruction of such defects. Lack of microsurgical team/setup or patient contraindication to undergo a lengthy procedure would divert the decision to local tissues for coverage typically using local fasciocutaneous/propeller flaps or muscle flaps. Also, those local flaps options would be the first choice for relatively small defects, with plenty of healthy tissue available for coverage. If a local fasciocutaneous flap is not feasible or desired, the distally based medial hemisoleus flap is a common reliable alternative for reconstruction of middle and/or distal third leg defects of small to moderate size. In the current series, relatively larger defects were successfully reconstructed using this flap. Intraoperative confirmation of the adequacy of the explored flap distal pedicle underly the reported satisfactory flap survival rate.
\end{abstract}

Patients and Methods: The study enrolled 13 cases (12 male \& 1 female) with distal and/or middle third leg defects. Patients' age ranged from 12-51 years age (mean 37 years), with defect sizes ranging from $3.5 \times 5 \mathrm{~cm}\left(17.5 \mathrm{~cm}^{2}\right)$ to $8 \times 10 \mathrm{~cm}$ $\left(80 \mathrm{~cm}^{2}\right)$ with a mean of $41 \mathrm{~cm}^{2}$. Distally based medial hemisoleus flap was used to reconstruct all cases, based on its distal pedicle(s) from the posterior tibial vessels. A split thickness skin graft was used to cover the muscle typically one week later in a second procedure.

Results: All flaps survived completely except partialthickness surficial flap necrosis in one case. This was conservatively managed by debridement and later skin grafting. All cases achieved complete healing of their wounds with successful limb salvage.

Conclusions: Intraoperative confirmation of distal medial hemisoleus flap pedicle would ensure better flap survival and would allow safe reconstruction of moderately large distal leg defects in selected cases with appropriate distal pedicle size and location.

Key Words: Medial hemisoleus flap - Distal leg defects Distally based flap.

\section{INTRODUCTION}

Distal leg traumatic injuries with open tibial fractures are common injuries. Reconstruction of such defects is a real challenge, and free tissue transfer is a straightforward decision for such defects [1-3], but some patients are not candidates for such procedure, also many hospitals lack microsurgery setup, a situation that would divert the decision to local tissues for coverage including local fasciocutaneous/propeller flaps and local muscle flaps. Also, those local flaps options would be the first choice for relatively small defects, with plenty of healthy tissue available for coverage with ease [4-6]. Local fasciocutaneous flaps would provide similar tissue type and bulk and avoid the functional loss of muscle harvest. Those flaps are typically transferred as simple transposition for middle third defects and propeller flap for more distal leg defects. Extensive injury to the surrounding skin or lack of anatomically appropriate fasciocutaneous perforators would preclude their use. Local muscle flaps would be a valuable alternative in such situation. Also, muscle flaps are considered 1 st choice for defects presenting with dead space/ cavity and/or heavy infection. Being more malleable, with effective dead space-filling with better delivery of immunologically active cells and antibiotics to the wound with better tissue ingrowth and incorporation into the defect [7-9], but more recent clinical data debated the superiority of muscle flaps compared to fasciocutaneous flaps in contaminated/infected beds in terms of limb salvage [10-12]. Local muscle flaps commonly used for distal leg defects include the medial hemisoleus muscle flap for middle and/or distal third leg defects of small to moderate size [13] and distally based peroneus brevis muscle flap for small distal leg defects [14].

The medial hemisoleus flap was first described by Tobin [13] in 1985. He described its use as a proximally based or distally based flap on its proximal or distal pedicles from the posterior tibial vessels, respectively. As a distally based flap, it 
could be used to cover small to medium-sized defects in the distal and middle third of the leg. A lot of publications [15-20] reported its successful use for this purpose with good functional outcome in defects up to $60 \mathrm{~cm}^{2}$. As a proximally based flap coverage of the distal third of the leg is typically limited to small-sized defects, as distal muscle bulk is typically small with significant variability. More proximal defect location over the middle leg third would improve the utility of the proximally based hemisoleus, yet the distally based variant is more advantageous for middle leg defects by transferring the bulkier proximal muscle segment for defect reconstruction, enabling coverage of relatively larger defects after confirmation of a reliable distal pedicle.

This retrospective analysis aims to report the author's experience with distally based medial hemisoleus flap for reconstruction of middle and/or distal third leg defects and its potentials for coverage of relatively larger distal leg defects up to $80 \mathrm{~cm}^{2}$.

\section{PATIENTS AND METHODS}

Between Jan 2012 to August 2017, 13 patients (12 males and 1 female) suffering from distal and/or middle third leg defects with exposed tibia were reconstructed with a distally based medial hemisoleus flap. Patients' age ranged from 12-51 years age (mean 37 years), with defect sizes ranging from $3.5 \times 5 \mathrm{~cm}\left(17.5 \mathrm{~cm}^{2}\right)$ to $8 \times 10 \mathrm{~cm}\left(80 \mathrm{~cm}^{2}\right)$ with a mean of $41 \mathrm{~cm}^{2}$.

Defect locations included the distal third of the leg in 5 patients, distal/middle third junction in 6 patients, and middle third in 2 patients. All were reconstructed with a distally based medial hemisoleus muscle flap. 12 out of the 13 cases were suffering from traumatic leg defects with underlying tibial fracture (Gustilo IIIB open tibial fracture) temporary fixed by an external fixator. In one case a middle third tibial defect was secondary to soft tissue sarcoma excision. 5 cases were reconstructed immediately after initial bone stabilization by external fixator or sarcoma excision. In 8 cases with a high risk of wound contamination and/or tissues of questionable viability, reconstruction followed initial bone stabilization by an average of 2 weeks during which wounds were managed by frequent dressing and debridement.

Pre-operative documentation included patient demographic data, cause, and size of the defect, and pre-operative photographs. No pre-operative angiography or doppler was done. Informed consent was taken from all cases.

\section{Surgical technique:}

Surgery was performed in a supine position with the affected lower limb abducted and externally rotated with slight knee flexion. All cases were operated on a tourniquet applied to the thigh with tourniquet pressure of $250 \mathrm{~mm} \mathrm{Hg}$ in children and $350 \mathrm{~mm} \mathrm{Hg}$ in adults.

Surgery started after liberal wound cleansing and irrigation with saline. Then wound debridement to healthy viable tissue edges was performed as indicated. Initial dissection was always done through the medial edge of the defect itself, to approach the distal medial soleus muscle/Achilles tendon, where careful blunt dissection and gentle retraction of the soleus muscle exposed the underlying deep muscle compartments and the posterior tibial vessels covered with deep leg fascia. Now the distal flap pedicle(s) from the posterior tibial vessels were visualized perforating the deep leg fascia to the medial hemisoleus muscle. A very limited longitudinal incision distally, or proximally would be added as needed for safe exposure in this stage. Now the distal pedicle is assessed regarding its appropriateness to nourish the desired flap size/length, before proceeding with further skin incision and flap harvest. Pedicle(s) size and location (flap pivot point) were the points to be assessed. At least one intact appropriately located perforator of approximately $1 \mathrm{~mm}$ or more with 2 accompanying veins, or one large vein must be confirmed before flap harvest, otherwise, a possible alternative flap would be planned.

Further exposure was performed by a longitudinal incision, $1.5 \mathrm{~cm}$ posterior to the medial border of the tibia, from the upper limit of the defect distally and extended proximally to the extent that would allow harvest of the desired flap length.

The proximal saphenous vein and nerve were carefully preserved even if the defect/trauma sacrificed segment of them. The incision was deepened through deep facias to expose the media head of gastrocnemius muscle proximally and the Achilles tendon and soleus muscle distally. Blunt dissection was used to separate the medial gastrocnemius from underlying medical hemisoleus in the relatively avascular plane in-between. Sharp dissection was used to release the proximal medial head of the soleus (medial hemisoleus) from its tibial origin while carefully protecting the underlying posterior tibial neurovascular bundle. One or more pedicles 
were encountered during this dissection from the posterior tibial vessels to the proximal medial hemisoleus, those were carefully ligated and divided, then the medial hemisoleus along with the mid muscle raphe was divided proximally at the desired level and dissected from the lateral head by a combination of sharp dissection and electrocautery.

The medial hemisoleus muscle was inset to the defect without tension by 3-0 half-buried absorbable mattress suture.

\section{Post-operative care:}

Typically, the flap was monitored every 4-6 hours during the $1^{\text {st }} 24$ hours post-operatively, then daily with daily dressing change. A split-thickness skin graft was then applied after one week when the flap viability was confirmed, or later after the management of any flap loss. The limb was immobilized with a below-knee slab and kept elevated; this was maintained for at least 3 weeks from the time of the flap harvest ( 2 weeks after application of the skin graft). Further immobilization was determined by the orthopedic surgeons as part of the management of associated tibial fractures.

\section{RESULTS}

A total of 13 patients ( 12 males \& 1 female) with distal and/or middle third tibial defects reconstructed by distally based medial hemisoleus flap were enrolled in this study. Follow-up period ranged from 6-18 months (mean 10 months).

Flap survived completely in 12 out of 13 patients $(92.3 \%)$. Only one case $(7.7 \%)$ suffered minor superficial flap sloughing. It was very superficial and did not result in underlying bone exposure, managed by debridement and later splitthickness skin grafting (Fig. 2). There were no reported cases of intraoperative neurovascular injury to the posterior tibial bundle, post-operative infection, or hematoma.

A split-thickness skin graft was applied one week after the flap harvest in a $2^{\text {nd }}$ procedure, except in one case with a relatively small flap that was grafted immediately. In the case with superficial flap sloughing, flap debridement and repeated dressing permitted skin grafting of healthy granulating bed 3 weeks post-operative. Table (1) represents a summary of patients' demographic data and results.

Table (1): Summary of patients' demographic data and results.

\begin{tabular}{|c|c|c|c|c|c|c|c|c|}
\hline Pt. & Age & Sex & Size/cm & Defect location & $\begin{array}{l}\text { Defect } \\
\text { etiology }\end{array}$ & $\begin{array}{l}\text { Timing of } \\
\text { reconstruction }\end{array}$ & Follow-up & $\begin{array}{l}\text { Flap } \\
\text { survival }\end{array}$ \\
\hline 1 & $14 y$ & Male & $5 \times 9 \mathrm{~cm}$ & Distal third & RTA & Delayed & 9 months & Complete \\
\hline 2 & $51 \mathrm{y}$ & Male & $5 \times 7 \mathrm{~cm}$ & Distal/middle third junction & RTA & Delayed & 7 months & Complete \\
\hline 3 & $33 y$ & Male & $4 \times 7.5 \mathrm{~cm}$ & Distal/middle third junction & RTA & Immediate & 6 months & Complete \\
\hline 4 & $28 y$ & Male & $5 \times 7 \mathrm{~cm}$ & Distal/middle third junction & RTA & Delayed & 12 months & Complete \\
\hline 5 & $47 y$ & Male & $8 \times 10 \mathrm{~cm}$ & Distal third & RTA & Immediate & 18 months & Complete \\
\hline 6 & $40 y$ & Male & $5 \times 8 \mathrm{~cm}$ & Distal/middle third junction & RTA & Delayed & 6 months & $\begin{array}{c}\text { Superficial } \\
\text { necrosis }\end{array}$ \\
\hline 7 & $35 y$ & Male & $5 \times 7 \mathrm{~cm}$ & Distal third & RTA & Delayed & 12 months & Complete \\
\hline 8 & $43 y$ & Male & $5 \times 6 \mathrm{~cm}$ & Distal third & RTA & Immediate & 8 months & Complete \\
\hline 9 & $31 \mathrm{y}$ & Male & $3.5 \times 5 \mathrm{~cm}$ & Middle third & RTA & Delayed & 6 months & Complete \\
\hline 10 & $27 y$ & Male & $6 \times 7 \mathrm{~cm}$ & Distal/middle third junction & RTA & Delayed & 10 months & Complete \\
\hline 11 & $12 \mathrm{y}$ & Female & $5 \times 8 \mathrm{~cm}$ & Distal third & RTA & Immediate & 13 months & Complete \\
\hline 12 & $31 \mathrm{y}$ & Male & $4.5 \times 7 \mathrm{~cm}$ & Distal/middle third junction & RTA & Delayed & 9months & Complete \\
\hline 13 & $39 y$ & Male & $8 \times 9 \mathrm{~cm}$ & Middle third & Sarcoma & Immediate & 11 months & Complete \\
\hline
\end{tabular}



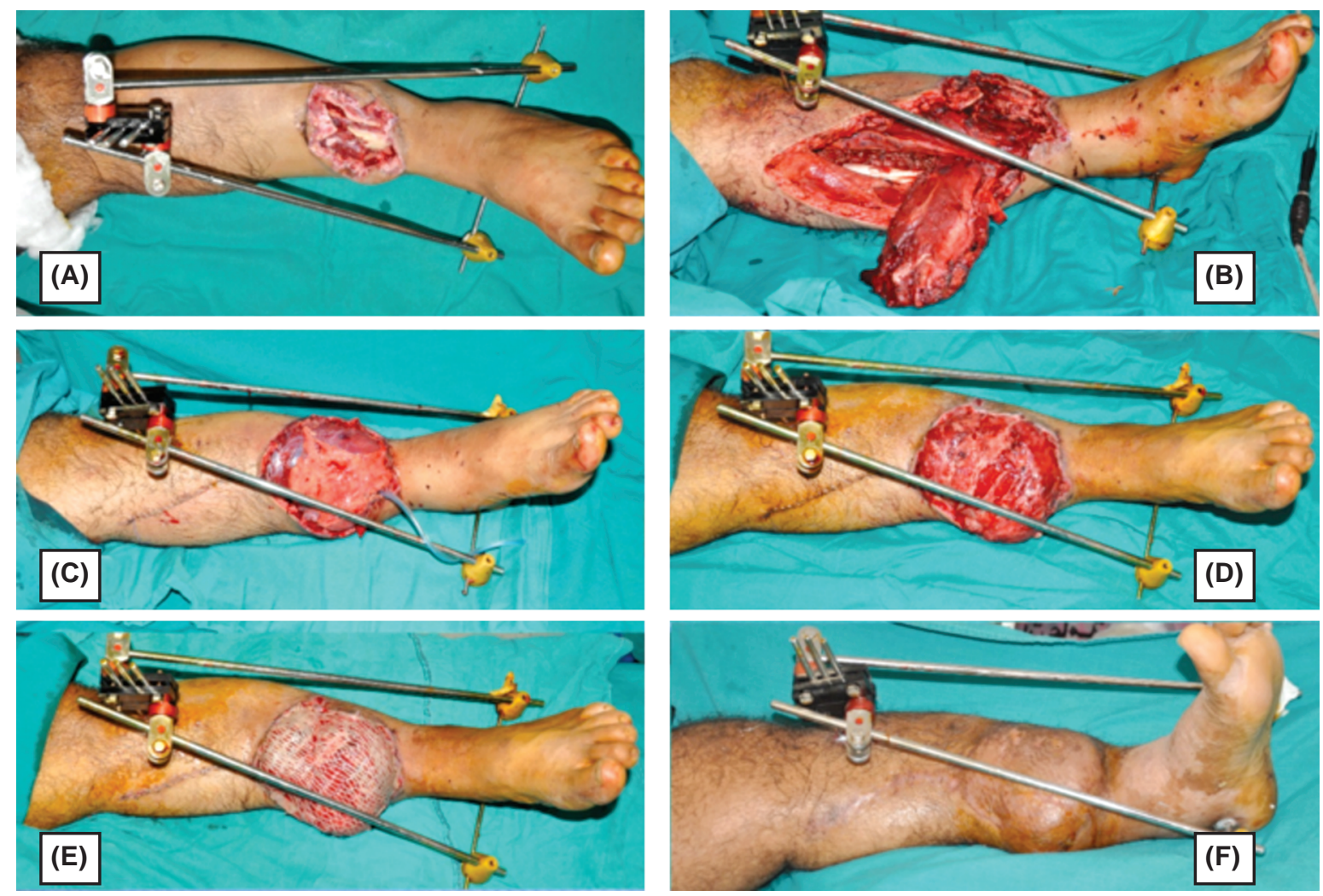

Fig. (1): 47y old male patient with Gustilo IIIB open distal tibial fracture. (A) After application of an external fixator by orthopedic surgeons, a distal third leg defect is seen before the required minimal edge debridement. (B) After minimal wound debridement and harvest of distally based medial hemisoleus flap. (C) Flap inset to the defect. (D \& E) oneweek later flap was fully viable before \& after split-thickness skin graft application. (F) Fully healed flap and skin graft 6 months later.
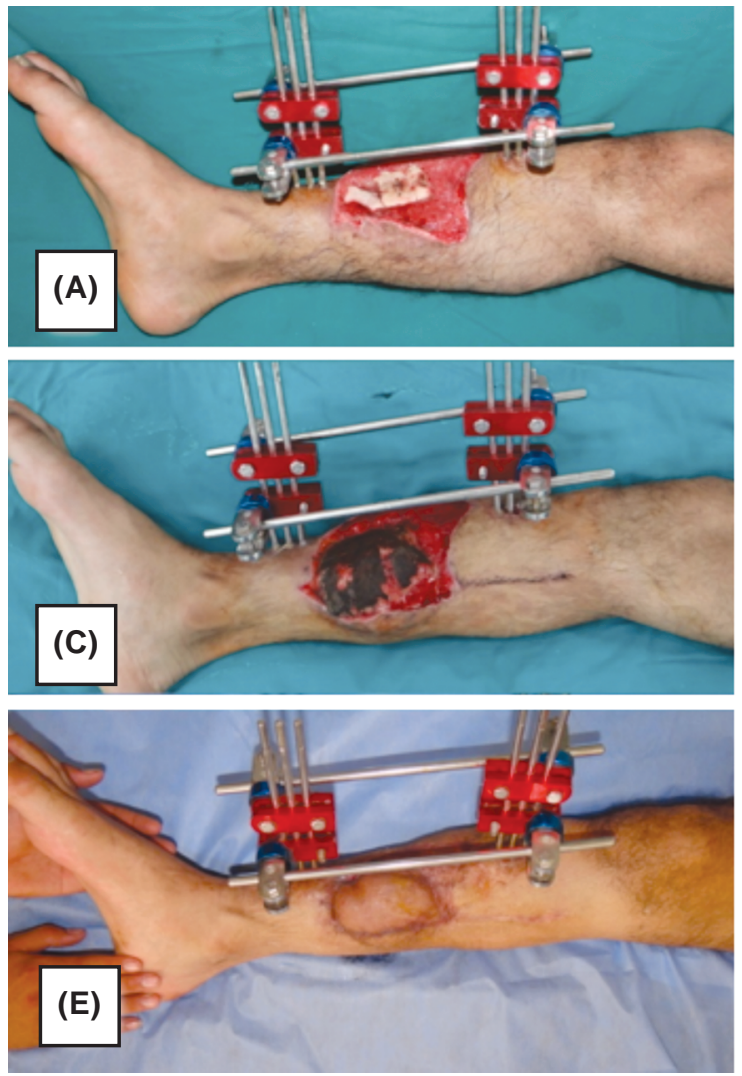
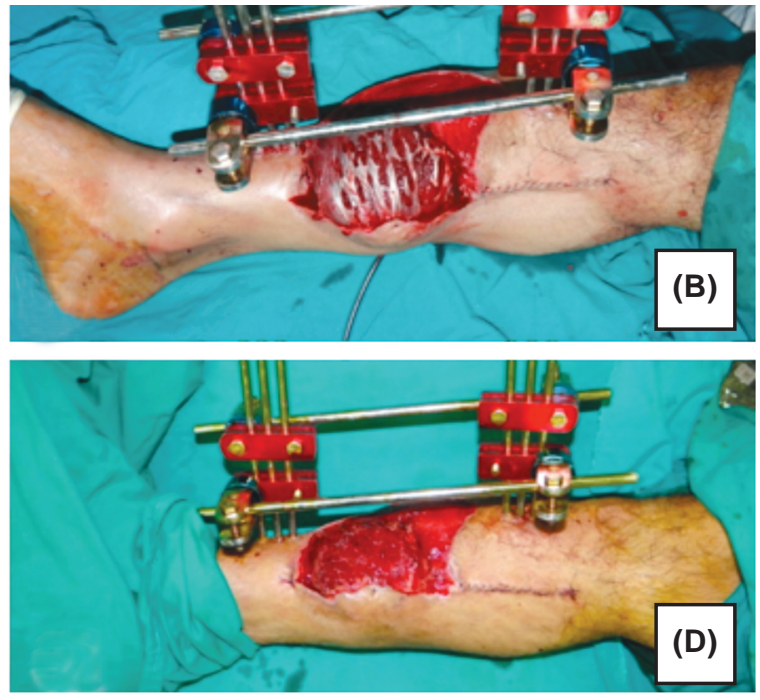

Fig. (2): 40y old male patient with Gustilo IIIB open distal tibial fracture. (A) After application of external fixator by orthopedic surgeons showing middle and proximal-distal third leg defect about $5 \times 8 \mathrm{~cm}$. (B) Flap inset to the defect, the aponeurotic muscle surface is partially excised to allow flap expansion and later rapid granulation. (C) One-week postoperative, flap showing superficial necrosis. (D) 2 weeks postoperative after debridement and immediately before splitthickness skin graft application. (E) Fully healed flap and skin graft 3-month post-operative. 

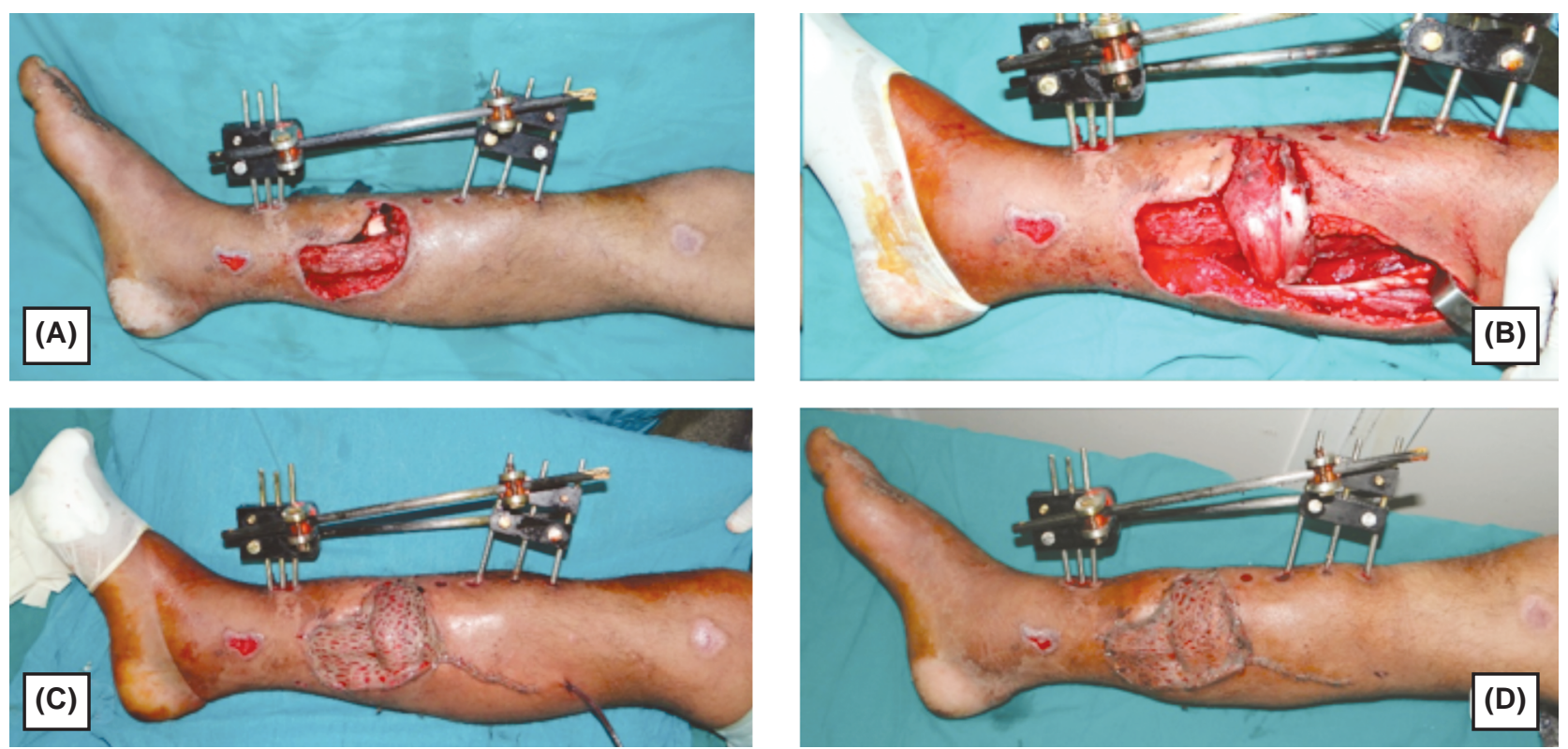

Fig. (3): 31 y male patient with a distal mid tibial defect. (A) 2 weeks post-trauma and fixation showing exposed fractured tibia with a defect for flap reconstruction of about $3.5 \times 5 \mathrm{~cm}$ and adjacent raw area over flexor digitorum longus and distal soleus muscles for grafting. (B) Distally based hemisoleus flap applied based on the most proximal of the distal perforators. (C) Meshed split-thickness skin graft applied in the same procedure. (D) One week later with full flap survival and graft take.
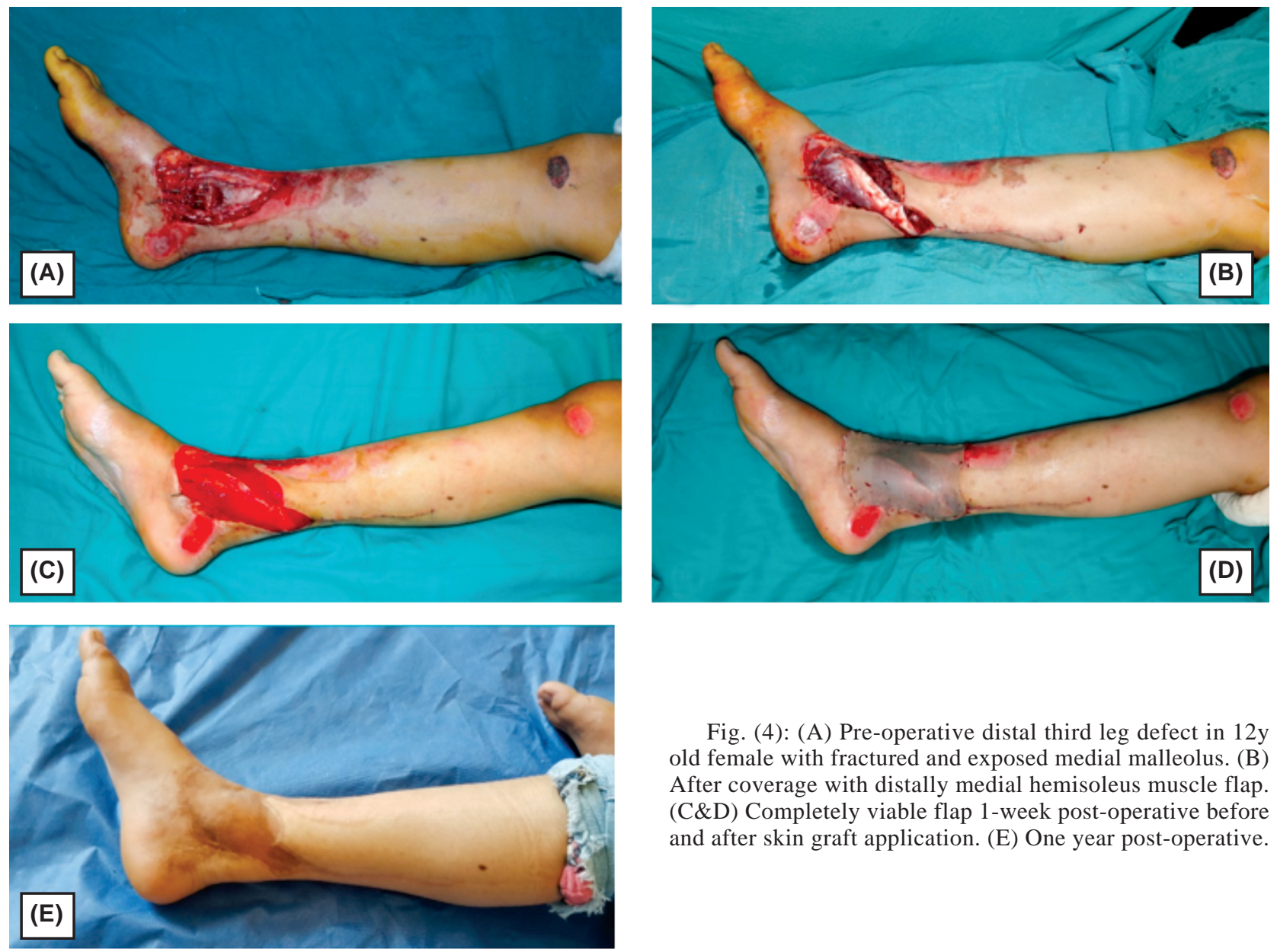

Fig. (4): (A) Pre-operative distal third leg defect in $12 \mathrm{y}$ old female with fractured and exposed medial malleolus. (B) After coverage with distally medial hemisoleus muscle flap. (C\&D) Completely viable flap 1-week post-operative before and after skin graft application. (E) One year post-operative. 


\section{DISCUSSION}

The soleus muscle is a bipennate muscle with a medial and lateral half connected by midline raphe with independent neurovascular supply for each half, permitting harvest of a medial hemisoleus flap without disturbing the lateral hemi-muscle function as first reported by Tobin [13] in 1985. He described its use as proximally or distally based flap, based on its proximal or distal pedicles from the posterior tibial vessels, respectively. Bulky reconstruction and loss of muscle function are the main drawbacks of using muscle flaps in general and this flap is not an exception, yet retention of meaningful ankle plantar flexion and near-normal gait is a clear advantage of hemisoleus flap, besides a better arc of rotation compared to complete muscle harvest [13].

It is a type 2 muscle flap based Mathes and Nahai [20] classification, being supplied by proximal main pedicle from the posterior tibial vessels and many minor distal pedicles from the same vessel [21]. Raveendran and Kumaragama [22] provided a more detailed description of soleus blood supply in a study of 50 cadavers dissections. They found an average of 5.4 perforators from the posterior tibial vessels (distal to the origin of peroneal vessels) to the medical hemisoleus along its whole length, of those an average of 3 perforators were found supplying the distal half of muscle in most cases, the most distal of those was $6.5 \mathrm{~cm}$ from medial malleolus on average. This rich distal blood supply is the basis for a distally based medial hemisoleus muscle. Unfortunately, this distal blood supply is not invariable, in the same study Raveendran and Kumaragama [22] reported $8 \%$ of cases collectively having either minimum of 3 perforators only or more than 7 perforators to the whole length of the medial soleus, without specifying the relative percentage of each. Confirmation of muscle/flap distal pedicle(s) is thus recommended pre and/or intraoperative before flap harvest to improve flap survival rate [16-19].

A distally based medial hemisoleus flap is indicated to cover middle and/or distal third leg defects of small to moderate size where free tissue transfer is not desired/not feasible [13,20-24]. Fasciocutaneous/propeller flaps are alternative flap options in such cases and represent the author's first choice, being of no functional morbidity and offer a reconstruction with similar tissue type and bulk. An extensive injury/raw area extending to nearby skin (fasciocutaneous flap territory) or inappropriate fasciocutaneous perforator anatomy revealed during initial surgical exploration preclud- ed the use of fasciocutaneous/propeller flaps for reconstruction of leg defects presented in this series and promoted the use of a distally based medial hemisoleus flap to reconstruct 13 cases of distal and/or middle third leg defects of small $(3.5 \times 5 \mathrm{~cm})$ to moderately large $(8 \times 10 \mathrm{~cm})$ size. All flaps survived completely except one case $(7.7 \%)$ of superficial flap necrosis that was managed conservatively with debridement and later skin grafting, with eventual complete healing in all cases. Flap distal pedicle(s) was surgically explored and confirmed initially before flap harvest in all cases. This probably underly the high flap survival rate encountered even with flap larger than reviewed literature reports [15-20]. No perioperative vascular studies (angiography) were done in any case, as this was not readily available and intraoperative pedicle confirmation was considered more practical and more accurate alternative. The reasonably reliable distal flap pedicle and possible alternative flap options planed were also other reasons to rely solely on surgical pedicle exploration without preoperative vascular study. Deserving mention, apart from the 13 reported flaps, in one case with distal leg defect initial exploration of the distal flap pedicle revealed inappropriate perforator anatomy, and the case was managed by free tissue transfer anastomosed to the explored posterior tibial vessels.

The peri or intraoperative confirmation of the flap pedicle was reported by other groups [9-11], almost with a similar success rate. Schierle et al. [16], studied a series of 17 patients reconstructed by distally based medial hemisoleus flap and reported complete flaps success with only one case $(6 \%)$ of partial flap tip necrosis. Besides pedicle confirmation, their success was also attributed to limiting flap length to the territory of the proximal ligated perforator just proximal to the flap distal pedicle perforator assuming that the blood flow from the latter would be carried to its proximal territory through choke vessels connecting both (angiosome concept). Typically, the flap tip was about $2-3 \mathrm{~cm}$ proximal to the entry point of this ligated proximal perforator.

In a series of 10 cases, Houdek and colleagues [17] performed a preoperative CT Angio (unless contraindicated) to assess the posterior tibial vessels pedicle(s) to the distal muscle, they also performed interoperative confirmation of the pedicle before flap harvest. They recommended a caliber of $1 \mathrm{~mm}$ minimum for the distal arterial perforator and a caliber of $1.5 \mathrm{~mm}$ or more for the accompanying vein to ensure flap reliability. They reported successful reconstruction in all cases, with only minor flap tip loss in one patient. Deserving mention, 
repeat skin grafting was needed in 4 out of the 10 cases in Houdek and colleague's series, an issue that was avoided in the current series, by delaying the skin grafting for one week after the flap harvest. This would prevent potential skin graft loss secondary to any possible flap loss, or simply because of the compromised early flap blood flow in a clinically viable flap. Delaying the skin graft allowed easier flap monitoring, ensured later grafting on a bed with better vascularity, and less exudation. Partial excision of the relatively avascular aponeurotic muscle surface after flap inset ensured later well-vascularized granulated bed. Only one case in this series received immediate skin grafting to avoid the main drawback of later grafting: The need for reoperation. In this case, the relatively small flap size, with a pivot point at mid-leg ensured rich flap vascularity, flap, and graft survival was completely successful.

Unluer and colleagues [18] reported an almost similar succusses rate in a report of 31 cases reconstructed by distally based hemisoleus, with only 2 cases suffered partial flap tip necrosis, they ensured capturing the distal 2 perforators to ensure flap vascularity, a measure that would explain this high success rate.

Rabbani and colleagues [19] reported a 37-case series of a distally based medial hemisoleus flap, with nearly a total of $11 \%$ partial flap loss. A lower flap survival rate was reported by other groups, ranging from $17 \%$ in series by Park and colleagues [23] to $30 \%$ in series by $\mathrm{Pu}$ [24], who attributed the flap tip loss in 3 out of 10 cases to heavy smoking or relatively large defect $\left(6 \times 10 \mathrm{~cm}=60 \mathrm{~cm}^{2}\right)$ in those cases. In the current series, the largest defect of $80 \mathrm{~cm}^{2}$ affecting the distal third of the leg was successfully reconstructed with a similarly large medial hemisoleus flap nourished by a large distal perforator (Fig. 1). This would reflect the importance of initial distal pedicle exploration before formal flap harvest, ensuring its adequacy for the desired flap size. In the current report, this allowed reconstruction of relatively larger defects $\left(72 \mathrm{~cm}^{2}\right.$ $\& 80 \mathrm{~cm}^{2}$ ) in selected cases with favorable anatomy expanding the typical flap/defect size limit of 50$63 \mathrm{~cm}^{2}$ reported in the literature $[\mathbf{1 5 - 1 9 , 2 3 , 2 4 ]}$. Such cases with relatively large defects should be approached with an alternative plane always ready. For example, the same initial pedicle dissection would allow exploration of skin perforators and provide recipient vessel preparation for possible free flap transfer.

As a proximally based, the hemisoleus muscle flap could be used to cover middle third leg defects, for more distal defects, including the distal third- middle third junction and distal third defects, its utility become limited, as the distal muscle bulk is markedly reduced with significant variability, and at the best, it could be used to reconstruct small defects depending on the available distal muscle bulk and its distal reach [24]. Even for middle third leg defects, the distally based medial hemisoleus conveying the larger proximal muscle segment would cover a larger defect compared to its proximal variant, provided that its distal pedicle(s) is intact and of adequate size. Initial exploration of the distal medial hemisoleus pedicle serves also to explore the distal muscle fleshy part (it's bulk and where it ends), which is an important determinant of the utility of the muscle as a proximally based flap for distal leg defects. In all cases of this series, the distal soleus muscle bulk was always too small and/or too proximal to reconstruct the presented defects.

\section{Conclusion:}

Intraoperative confirmation of distal medial hemisoleus flap pedicle would ensure better flap survival and would allow safe reconstruction of moderately large distal leg defects in selected cases with appropriate distal pedicle size and location. The flap is easy to harvest and has an acceptable donor site morbidity.

\section{REFERENCES}

1- Marek C.A. and Pu L.L..: Refinements of free tissue transfer for optimal outcome in lower extremity reconstruction. Ann. Plast. Surg., 52: 270-275, 2004.

2- Swartz W.M. and Mears D.C.: The role of free-tissue transfer in lower extremity reconstruction. Plast. Reconstr. Surg., 76: 364-373, 1985.

3- Heller L. and Levin L.S.: Lower extremity microvascular reconstruction. Plast. Reconstr. Surg., 108: 1029-1041, 2002.

4- Nazerali R.S. and Pu L.L.: Free tissue transfer to the lower extremity: A paradigm shift in flap selection for soft tissue reconstruction. Ann. Plast. Surg., 70: 419-422, 2013.

5- Pu L.L.: Soft-tissue reconstruction of an open tibial wound in the distal third of the leg: A new treatment algorithm. Ann. Plast. Surg., 58 (1): 78-83, 2007.

6- Thornton B.P., Rosenblum W.J. and Lee L.: Reconstruction of limited soft-tissue defect with open tibial fracture in the distal third of the leg. Ann. Plast. Surg., 54 (3): 27680, 2005.

7- Calderon W., Chang N. and Mathes S.J.: Comparison of the effect of bacterial inoculation in musculocutaneous and fasciocutaneous flaps. Plast. Reconstr. Surg., 77 (5): 785-94, 1986.

8- Chang N. and Mathes S.J.: Comparison of the effect of bacterial inoculation in musculocutaneous and randompattern flaps. Plast. Reconstr. Surg., 70 (1): 1-10, 1982. 
9- Gosain A., Chang N., Mathes S., et al.: A study of the relationship between blood flow and bacterial inoculation in musculocutaneous and fasciocutaneous flaps. Plast. Reconstr. Surg., 86 (6): 1152-63, 1990.

10- Salgado C.J., Mardini S., Jamali A.A., et al.: Muscle versus nonmuscle flaps in the reconstruction of chronic osteomyelitis defects. Plast. Reconstr. Surg., 118 (6): 1401-11, 2006.

11- Yazar S., Lin C.H., Lin Y.T., et al.: Outcome comparison between free muscle and free fasciocutaneous flaps for reconstruction of distal third and ankle traumatic open tibial fractures. Plast. Reconstr. Surg., 117 (7): 2468-75, 2006.

12- Cho E.H., Shammas R.L., Carney M.J., et al.: Muscle versus fasciocutaneous free flaps in lower extremity traumatic reconstruction: A multicenter outcomes analysis. Plast. Reconstr. Surg., 141 (1): 191-9, 2018.

13- Tobin G.R.: Hemisoleus and reversed hemisoleus flaps. Plast. Reconstr. Surg., 76: 87-96, 1985.

14- Hassan K.M., Mohamed A.M. and Mohamed M.S.: Clinical Evaluation of Peroneous Brevis Muscle Flap as a Reconstructive Tool for Defects Around the Ankle. Egypt, J. Plast. Reconstr. Surg., 43 (3): 557-564, 2019.

15- Pu L.L.: The reversed medial hemisoleus muscle flap and its role in reconstruction of an open tibial wound in the lower third of the leg. Ann. Plast. Surg., 56: 59-64, 2006.

16- Schierle C.F., Rawlani V., Galiano R.D., et al.: Improving outcomes of the distally based hemisoleus flap: Principles of angiosomes in flap design. Plast. Reconstr. Surg., 123: 1748-1754, 2009.
17- Houdek M.T., Wagner E.R., Wyles C.C., et al.: Reverse medial hemisoleus flaps for coverage of distal third leg wounds: A technical trick. J. Orthop. Trauma, 30 (4): 138$42,2016$.

18- Unluer Z., Al-Ajam Y. and Al-Benna S.: Functional outcome after reconstruction of traumatic soft tissue defects in the lower half of the leg with distally based medial hemisoleus muscle flaps. A case series and literature review. Ann. Plast. Surg., 81: 468-471, 2018.

19- Rabbani M.J., Ata-ul-Haq, Riaz A., et al.: Distally based medial hemisoleus flap: reliable option for distal tibial wounds. J. Coll Physicians Surg. Pak., 28 (2): 129-32, 2018.

20- Mathes S.J. and Nahai F.: Classification of the vascular anatomy of muscle: Experimental and clinical correlation. Plast. Reconstr. Surg., 67: 177-87, 1981.

21- Hallock G.G.: Getting the most from the soleus muscle. Ann. Plast. Surg., 36: 139-146, 1996.

22- Raveendran S.S. and Kumaragama K.G.: Arterial supply of the soleus muscle: Anatomical study of fifty lower limbs. Clin. Anat., 16: 248-252, 2003.

23- Park I.J., Sur Y.J. and You S.L.: Reconstruction of the soft tissue defect on anteromedial surface of the leg using medial hemisoleus flap. J. Korean Soc. Microsurg., 23:76$81,2014$.

24- Pu L.L.: Further experience with the medial hemisoleus muscle flap for soft-tissue coverage of a tibial wound in the distal third of the leg. Plast. Reconstr. Surg., 121: 2024-2028, 2008. 